\title{
Textiles: The Future
}

\author{
Michael P Londrigan* \\ LIM College, USA
}

*Corresponding author: Michael P Londrigan, Vice President for Academic Affairs, LIM College, New York, USA.

Received Date: December 04, 2018

Published Date: December 06, 2018

\section{Introduction}

It is often said in the world of fashion that fashion is "evolutionary not revolutionary". I agree with that statement that fashion is evolutionary but only from a design and silhouette standpoint. Today the industry is truly revolutionary from a textile standpoint. It is therefore my opinion that the statement as written needs to have a new chapter added where fashion becomes revolutionary and evolutionary at the same time with textiles leading that revolutionary.

For those that have grown up in the fashion business where fiber choice was limited to several mainstays such as natural fibers like cotton, wool, linen and silk and the man-made fibers like polyester and nylon it is refreshing to see what is happening through the use of technology and innovation in fiber and textile development. It is not only a revolution in new fibers and fiber creation but there are exciting strides that have been made in the mainstays mentioned above that has excited fiber aficionados from around the world. Through the use of improved seed development to unique spinning, dyeing and finishing techniques the fibers and textiles of today are far more interesting to those of the past.

The revolution is being led by both traditional non-traditional fiber and textile companies. Traditionalists such as Cotton Incorporated in conjunction with chemical and seed companies are creating bug tolerant strains of cotton while increasing staple length. As for the non-traditional companies I am highlighting several in this article and they all have common traits: not traditional fiber and textile producers, technology driven, venture capital funded, new to the fashion industry, sustainable, and exciting to follow.

\section{Bolt threads}

"We believe that answers to our most vexing problems can be found in nature. Every day we're inspired by the amazing materials we work with and driven by the desire to turn these materials into incredible products that solve the problems of a resourceconstrained world." Bolt has developed the technology to replicate spider silk for commercial use and has a line of knit ties to prove it.

\section{Orange fiber}

"We are the world's first and only brand to produce a patented material from citrus juice byproducts, repurposing them to create beautiful, sensorial materials that reshape your sartorial experience. Our fabrics are formed from silk-like cellulose yarn that can blend with other materials. When used in its purest form, the resulting $100 \%$ citrus textile features a soft and silky hand-feel and lightweight." Textiles, food and Italian knowhow coming together to produce a truly unique fiber/fabric.

\section{AlgiKnit}

Uses kelp, a type of seaweed, to produce a biopolymer called alginate which is then used for textile production. AlgiKnit produces a line of knitted sweaters form yarns developed by nature under water.

\section{Modern meadow}

A New Jersey-based company designs DNA that can yield collagen, the protein that makes up leather. These specially constructed DNA strands are inserted into yeast cells producing collagen and other proteins that are essential in forming leather. This process can be linked to lab grown leather like products. The tradename under which Modern meadow will be marketing their line of leather like products is Zoa.

\section{Mango materials}

Is a California start up that makes fabrics by feeding wastewater methane to methanotrophic bacteria that eat it and produce PHAbased polyester that can be woven into threads or molded into various shapes? Now that is truly revolutionary and a fine use of chemistry.

This is only a snap shot of where we are today and no doubt many reading this journal are familiar with these companies and what they are capable of. The point of the piece is to show just how revolutionary the fashion industry has become and where the future is going. Given the emphasis on sustainable sourcing, 
materials creation, and our current dependence on fibers that use large quantities of natural resources such as oil and water the industry must embrace the revolution and find ways to make these and new innovations commercially viable. I believe this is only the beginning and through technology and imagination the revolution in textiles and fiber creation in its infancy.

\section{Acknowledgement}

None.

\section{Conflict of Interest}

No conflict of interest. 